\title{
Optimization of Current Collector Design for Operando X-Band- EPR Investigations of Lithium-Ion Batteries using Numerical Simulations
}

\author{
Ivan Flammia ${ }^{1}$, Achim Mester ${ }^{1}$, Arvid Niemöller ${ }^{2}$, Josef Granwehr ${ }^{2,3}$ and Stefan van Waasen ${ }^{1,4}$ \\ ${ }^{1}$ Central Institute for Engineering, Electronics and Analytics — Electronic Systems (ZEA-2) \\ Forschungszentrum Jülich GmbH, Jülich, Germany \\ ${ }^{2}$ Institute of Energy and Climate Research — Fundamental Electrochemistry (IEK-9) \\ Forschungszentrum Jülich GmbH, Jülich, Germany \\ ${ }^{3}$ Institute of Technical and Macromolecular Chemistry (ITMC) \\ RWTH Aachen University, Aachen, Germany \\ ${ }^{4}$ Faculty of Engineering, Communication Systems (NTS) \\ University of Duisburg-Essen, Duisburg, Germany
}

\begin{abstract}
Monitoring the operation of lithium-ion batteries using electron paramagnetic resonance (EPR) is a powerful tool for gaining a better understanding of the redox processes and degradation mechanisms in anode and cathode materials. For this purpose, a complete operational cell, including current collectors, must be placed into the region of maximum magnetic field and minimum electric field of the specific resonance mode established in the EPR cavity. The homogeneity of the microwave (MW) magnetic field in the measurement region is essential to perform accurate quantitative measurements. Furthermore, the samples, the current collectors, and the dielectric holders should be confined within a region with negligible electrical field, to prevent unwanted power dissipation and to avoid a reduction of the cavity quality factor.

Current collectors represent a major problem in the EPR characterization of battery cells, as the introduction of conducting elements into the resonator affects the electromagnetic field distribution, thus altering the EPR spectra, or prevents their acquisition altogether.

Furthermore, the collectors should not excessively cover the battery surface, as this would shield the MW field at the position occupied by the active materials in the cell. On the other hand, they should be evenly distributed over the surface of the cell to prevent the establishment of transversal electrical gradients and guarantee a homogenous development of electrochemical processes.

In this paper, we discuss the simulation results for various circular current collector geometries with a diameter of $7 \mathrm{~mm}$, suitable for operando X-band-EPR measurements of battery cells in a Bruker Elexsys E540 spectrometer. A commercial 4108 TMHS cylindrical resonator is operated using the $\mathrm{TM}_{110}$ mode in order to excite and monitor the EPR resonances. The designs take into account practical manufacturability considerations: The collectors are specifically engineered to be easily integrated into the assembly, using
\end{abstract}


substrates which are EPR silent and chemically inert against the battery materials.

\section{INTRODUCTION}

Electron paramagnetic resonance (EPR) is a spectroscopy technique based on the interaction of a microwave (MW) electromagnetic (EM) radiation with unpaired electrons in materials. A simplified representation of an EPR reflection spectrometer is shown in Figure 1-a. The MW bridge contains a MW source and detector, as well as power supply, control and protection electronics, and an isolator. The MW signal is fed to the resonator cavity through an iris and the reflected signal, containing information about the electron-spin transitions occurring within the sample, is read by the detector. The generated and the reflected MW signals share the same transmission line (usually a rectangular waveguide) to the cavity, but are separated by a circulator within the MW bridge. The cavity is placed in an electromagnet, used to create a homogenous static magnetic field that is modulated by an auxiliary coil next to the cavity at frequencies below $1 \mathrm{MHz}$. The resulting signal represents the first derivative of the absorption EPR spectrum of the sample [1].

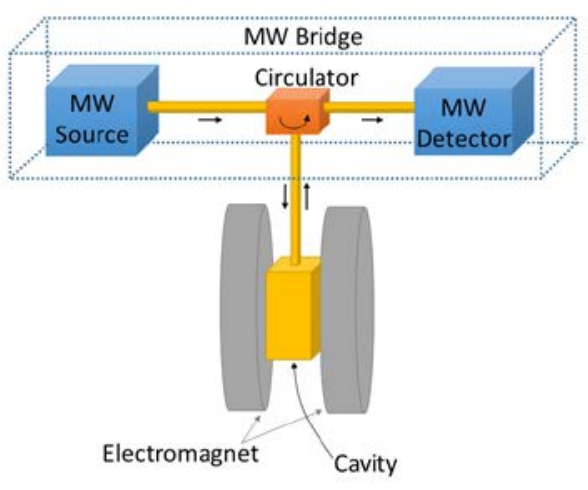

(a)

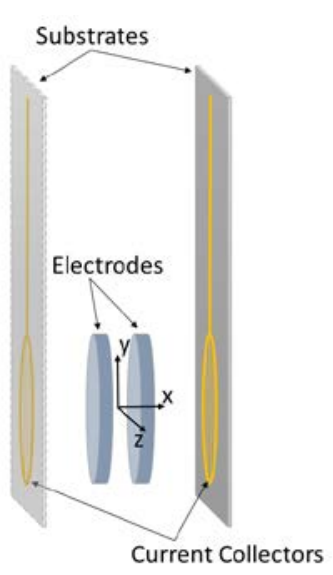

(b)

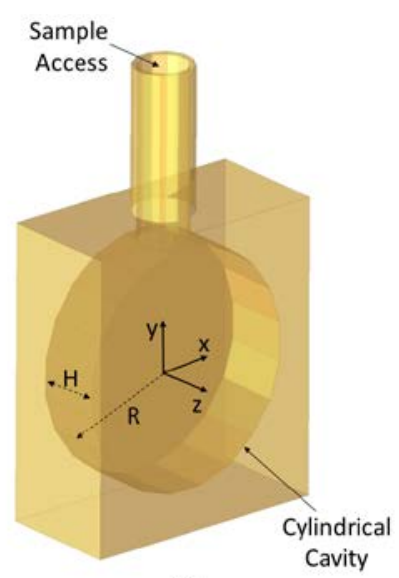

(c)

Figure 1: (a) Schematic representation of a continuous-wave EPR spectrometer. Power supplies, reference lines, and protection and control electronics have been omitted. (b) Simplified blow-up view of the battery cell assembly. The separator, the electrolytes and the quartz glass support are not shown. (c) Model of the 4108 TMHS resonator used for EM simulations. The cell will be oriented along the $y-z$

plane in the center of the cavity. The resonator is feed through a lateral iris from a rectangular waveguide that is not included in this model. The battery and the resonator are not represented to the same scale.

Operando EPR spectroscopy is a powerful technique to investigate redox processes and degradation mechanisms for anode and cathode materials of lithium-ion battery cells during charge/discharge cycling, where traditional in situ techniques fail at identifying crucial transient aspects of cell degradation [2, 3]. However, this technique requires that a complete operational cell (Figure 1-b), including current collectors, is placed into the resonator cavity, which sets additional constraints on the cell design and restricts the choice of materials for the cell assembly. They must be EPR silent and have minimal impact on the EM field used to excite the resonance, while being compatible with the used electrolytes. An even 
more critical problem is represented by the introduction of conducting elements, in particular the current collectors, into the resonator, as they will affect the distribution of the MW field around the sample, altering the total magnetic field acting on the sample's electrons and finally compromising the quality of the extracted EPR spectra.

As a result, only few papers have been addressing operando EPR analysis of battery cells to date [37] and, to the authors' knowledge, the impact of the current collectors on the quality of the measurement has never been assessed.

In this paper we discuss the design of current collectors suitable for X-band-EPR investigation of circular lithium-ion cells and their inclusion in the whole cell assembly.

\section{CELL ASSEMBLY AND RESONATOR MODEL}

The battery cells have a diameter of $8 \mathrm{~mm}$ and are assembled using a quartz glass housing [6]. A perfluoroalkoxy (PFA) sheet is used as substrate for the current collectors, as tests have shown that it is silent to EPR analysis and does not react when in contact with the aprotic electrolytes used in the cell [8]. The current collectors will be manufactured by depositing thin aluminum films (expected thickness $\sim 5 \mu \mathrm{m}$ ) onto the substrates by chemical vapor deposition (CVD).

The resonator in use is a Bruker 4108 TMHS cylindrical resonator, in which a TM110 mode is excited, which has the characteristic of having a uniform field distribution along the $z$-axis. Figure 1-c shows the simplified model of the resonator used for EM simulations. It essentially consists of an air-filled cylindrical cavity with radius $R=18.5 \mathrm{~mm}$ and height $H=11 \mathrm{~mm}$. The resonant frequency of the $\mathrm{TM}_{110}$ mode for this resonator can be estimated to be [9]

$$
f_{T M, 110}=\frac{1}{2 \pi \sqrt{\varepsilon_{0} \mu_{0}}} \sqrt{\left(\frac{p_{11}}{R}\right)^{2}} \approx 9.88 \mathrm{GHz},
$$

where $\varepsilon_{0}$ and $\mu_{0}$ are the vacuum permittivity and permeability, respectively, and $p_{11}$ is the first root of the first-order Bessel function of the first kind $\mathrm{J}_{1}\left(k_{c} \rho\right)$, with $k_{c}$ being the cut-off wave number of the resonance frequency and $\rho$ representing the radial coordinate in the $x-y$ plane of the coordinate system shown in Figure 1-c. The relative permittivity and the permeability of air have been assumed to be $\varepsilon_{\mathrm{r}} \approx \mu_{\mathrm{r}} \approx 1$. The theoretical value of the resonance frequency is close to the nominal $9.8 \mathrm{GHz}$ specified by the manufacturer [10]. The resonance frequency estimated using the eigenmode solver of the software Keysight EMPro is $9.92 \mathrm{GHz}$ and the corresponding electric and magnetic fields are shown in Figure 2. All eigenmode simulations reported in this paper are performed assuming an energy amount of $1 \mathrm{~J}$ initially trapped within the resonator.

The model depicted in Figure 1-c further includes a cylindrical aperture on the top side of the resonator, which is used to introduce the sample into the resonator and which is essential to model the complete current collectors, as shown in Figure 7-b. This feature has a negligible impact on the resonating mode, as it is located at a point of minimum field magnitude for the TM110 mode, as it can be seen by inspecting the field distribution in Figure 2.

Brass is used as material for modelling the metal body of the resonator, while air is used as dielectric 
for the cavity. Although the surface roughness could increase ohmic losses, no correction has been applied to take its impact into account, as the mean value of the surface roughness for the real resonator in use is not known. As a result, the $Q$ value of 5230 estimated by the simulation for the empty resonator (see Figure 7-c) might be higher than the actual one and should be considered as an ideal benchmark value.

To reduce undesired MW power dissipation, the sample and the current collectors of the battery should be confined within a region with negligible electrical field. The quartz support is not considered in this analysis, as its dielectric loss tangent is at least one order of magnitude lower than the one associated with PFA $\left(\tan \delta_{\mathrm{QUARTZ}} \sim 0.0001 \ll \tan \delta_{\mathrm{PFA}} \sim 0.001\right)$. By inspection of the E-field distribution in the $x-y$ plane (see Figure 3), it is noticed that there is a low-intensity field region in the center of the resonator. Within a distance of $350 \mu \mathrm{m}$ from the center, the magnitude of the $E$-field is less than $10 \%$ of the maximum value. This identifies a 700 - $\mu$ m-thick region in which the cell, the current collectors and the substrates can be placed without incurring excessive loss. In an effort to further minimize losses, we will assume a thickness of $300 \mu \mathrm{m}$ for the cell and $100 \mu \mathrm{m}$ for each PFA substrate, thus restricting the cell assembly to a region with a thickness of only $500 \mu \mathrm{m}$.

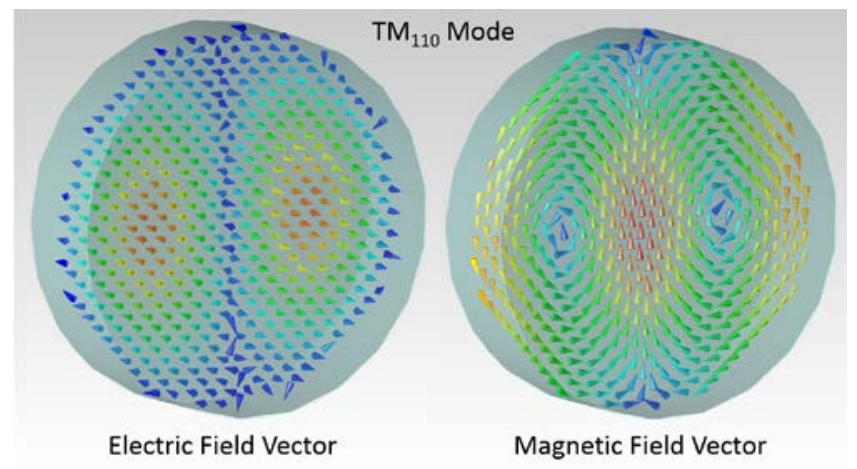

Figure 2: Electric and magnetic field vectors of the $\mathrm{TM}_{110}$ mode in a cylindrical resonator, plotted for the $z=0$ plane. The color of the arrows represents the intensity of the fields (red = strong, blue $=$ weak/zero). The field distribution is uniform along the $z$-axis.

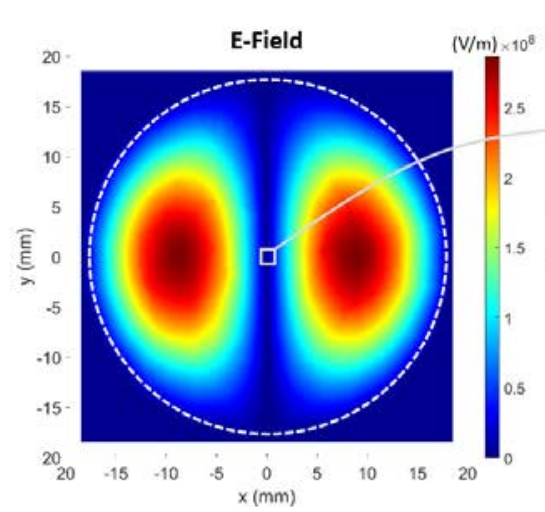

(a)

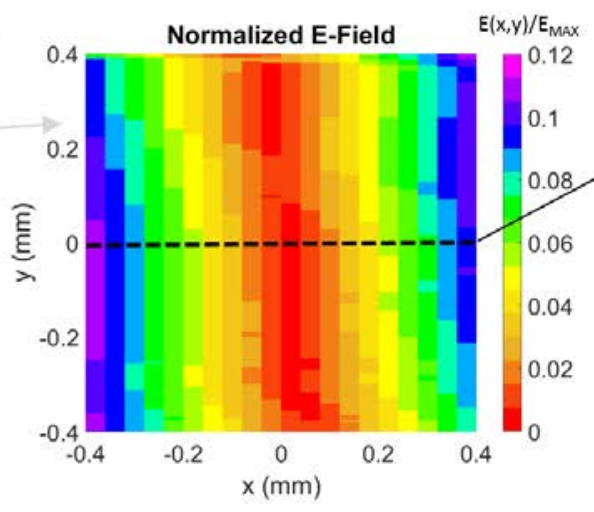

(b)

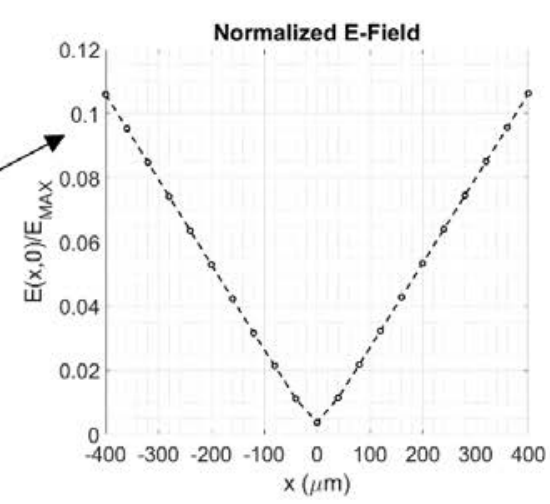

(c)

Figure 3: (a) Magnitude of the electric field in the $x-y$ plane within the whole resonator. The superimposed white dashed line identifies the contour of the cylindrical cavity. (b) Normalized electric field distribution $\left(\mathrm{E}(\mathrm{x}, \mathrm{y}) / \mathrm{E}_{\mathrm{MAX}}\right)$ within an area of $800 \times 800 \mu \mathrm{m}$. (d) Normalized electric field along the $y=0$ line (center of the resonator). 


\section{DESIGN OF THE CURRENT COLLECTORS}

The current collectors should be homogeneously distributed over the surface of the cell to prevent the occurrence of transversal electrical gradients and guarantee homogenous electric and ionic currents in the material. On the other hand, they should not excessively cover the cell surface, as this would shield the MW field within the cell. Therefore full discs or patches cannot be considered as a viable option, unless their thickness can be kept below the skin depth of the deposited metal (e.g. $\sim 0.8 \mu \mathrm{m}$ at $9.8 \mathrm{GHz}$ for aluminum), which is not achievable with the current manufacture process. Alternatively, grating-like structures may be used. Vertical gratings are preferred, as they are less prone to interact with the locally horizontal electric and locally vertical magnetic field vectors (visible in Figure 2). The vertical direction is specified by the $y$-axis of the coordinates system introduced in Figures 1-b and 1-c . In this paper, three circular configurations with 13 vertical connecting lines are evaluated (see Figure 4): one in which the vertical grating is connected to two quarter rings, one in which the vertical grating is connected to a half ring, and one in which the vertical grating is connected to a full ring. The pitch of the vertical grating is set to $500 \mu \mathrm{m}$. Although the cells have an 8-mm diameter (with implies a cell area of $\sim 50.3 \mathrm{~mm}^{2}$ ), the diameter of the current collectors has been chosen to be $7 \mathrm{~mm}$, to facilitate the cutting out into shapes of appropriate size for the housing in the quartz support.
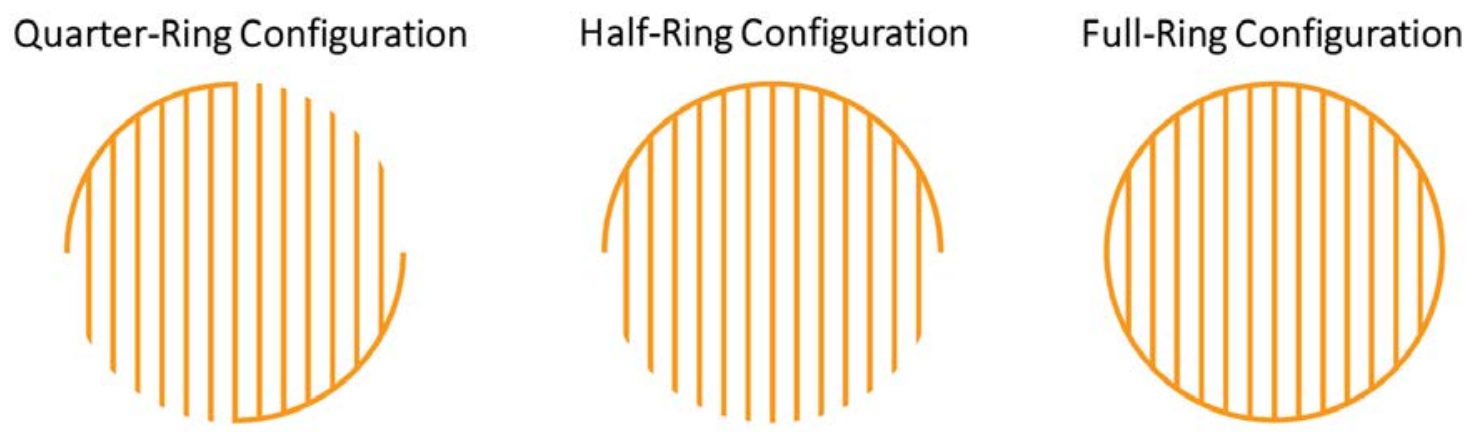

Figure 4: Proposed configurations for the current collectors.

The amplitude and the homogeneity of the total magnetic field in the measurement region are essential to perform sensitive and quantitative measurements. For this reason, the fluctuations of the MW magnetic field should be minimized in the area occupied by the cell. Figure 5 shows the histograms representing the distribution of the simulated magnitude of the MW magnetic field in the $y$-z plane, at the center of the cell $(x=0)$, for the three configurations of the current collectors, using a line width of $100 \mu \mathrm{m}$. For these simulations, a sandwiched version of each current collector configuration introduced in Figure 4 has been introduced in a simplified model of the resonator (a hollow brass cylinder without sample access). It can be seen that the histogram associated with the half-ring configuration has the smallest standard deviation $(\sigma)$, which indicates a better overall homogeneity of the field. At the same time, this configuration shows a high mean value of the $H$-field magnitude, which is a desired feature as it leads to higher measurement sensitivity. The mean value of the distribution of the quarter-ring configuration offers a comparable mean value but a considerably larger standard deviation, while the full-ring configuration has the smallest mean value, as well as the largest standard deviation. The plots 
of the homogeneity of the $H$-field with respect to the mean value, defined as $\left|\boldsymbol{H}(\mathrm{y}, \mathrm{z})-\boldsymbol{H}_{\mathrm{MEAN}}\right| /\left|\boldsymbol{H}_{\mathrm{MEAN}}\right|$ and shown in Figure 6 for each configuration, further confirm that the field values of the half-ring configuration have the least deviation from the mean value. Therefore, the half-ring configuration is the most appropriate design for the cell assembly considered in this paper.

However, as shown in Figures 5 and 6, the largest field deviations of the full-ring configuration occur close to the top and bottom edges (where the profile of the ring approximates horizontal lines), while the rest of the area shows a rather homogeneous magnitude distribution. This design is therefore only recommendable for cells with a diameter smaller than the one of the current collector ring.
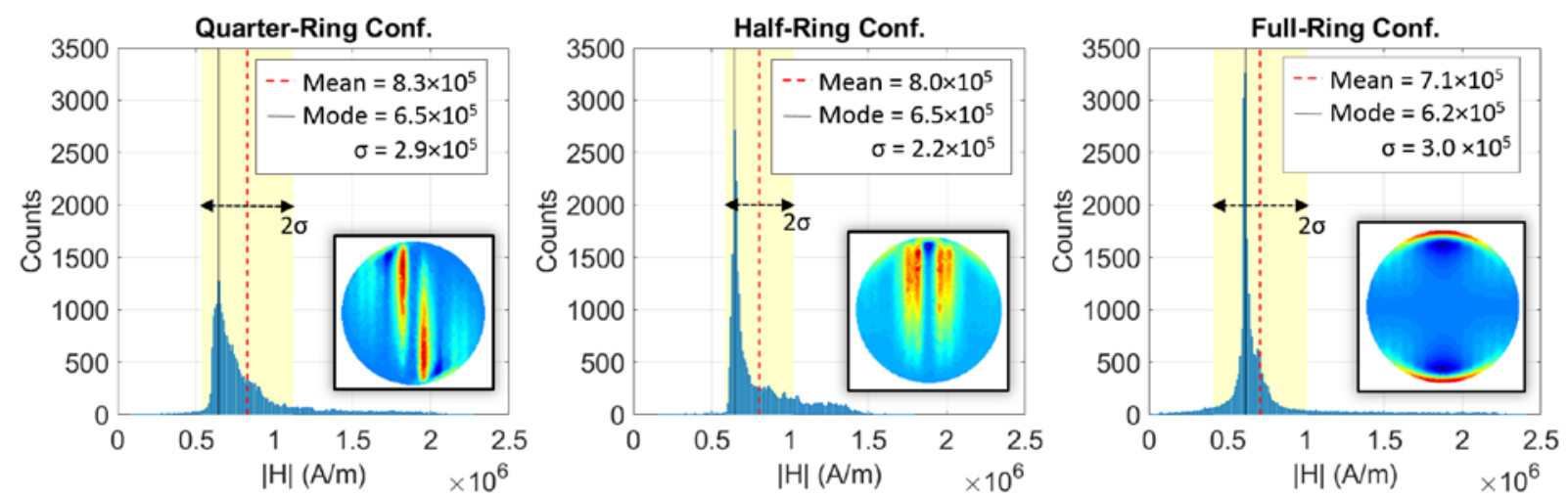

Figure 5: Histograms showing the counts of the MW $H$-field values in the $y$ - $z$ plane between the current collectors (at $x=0$ ) for the three configurations. The bin width is set to $10^{4} \mathrm{~A} / \mathrm{m}$. The yellow boxes have widths equal to twice the standard deviation $(\sigma)$ of the corresponding datasets. The insets show the corresponding qualitative $H$-field magnitude (red = strong field, dark blue = low field).
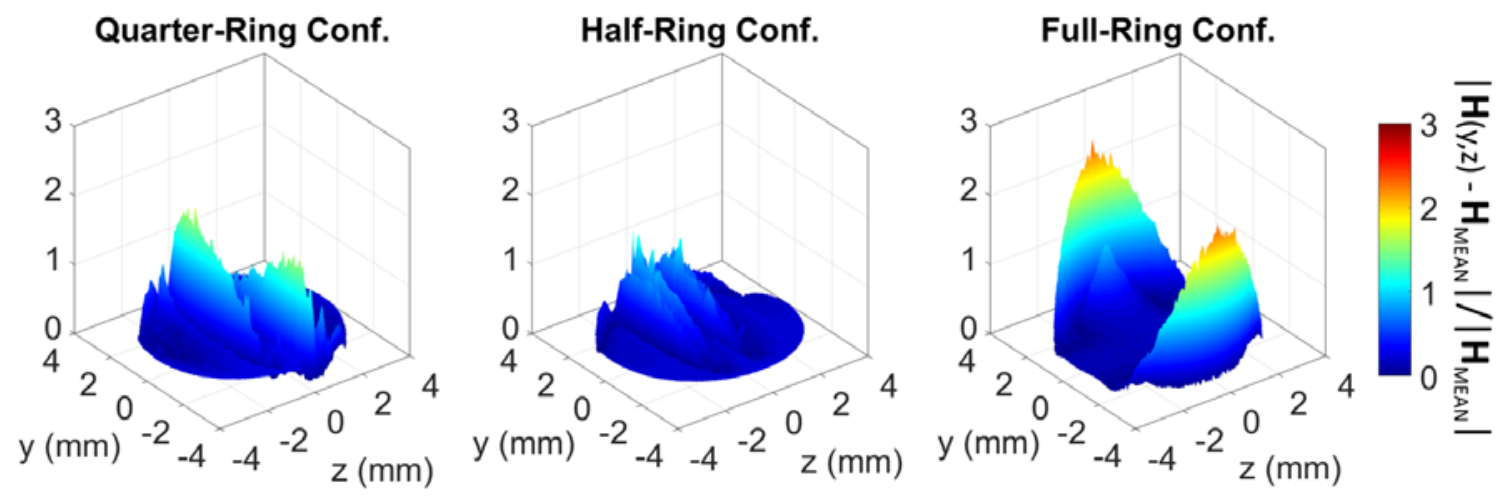

Figure 6: Homogeneity of the magnetic field between the current collectors (at $x=0$ ) for the three different configurations.

In order to evaluate the impact of the three configurations on the $Q$ value of the resonator, the long leads used to drain the DC current generated by the battery have been added to the current collectors (as shown in Figure 7-a), and the model of the resonator introduced in Figure 1-c has been adopted. Figure 7-b shows an example of the complete model used for the simulations, featuring the full-ring configuration. It can be seen from the values reported in Table 1, that the quarter-ring and the half-ring configurations are essentially equivalent, while the full-ring configuration has a stronger negative impact 
on the $Q$ value of the resonator.

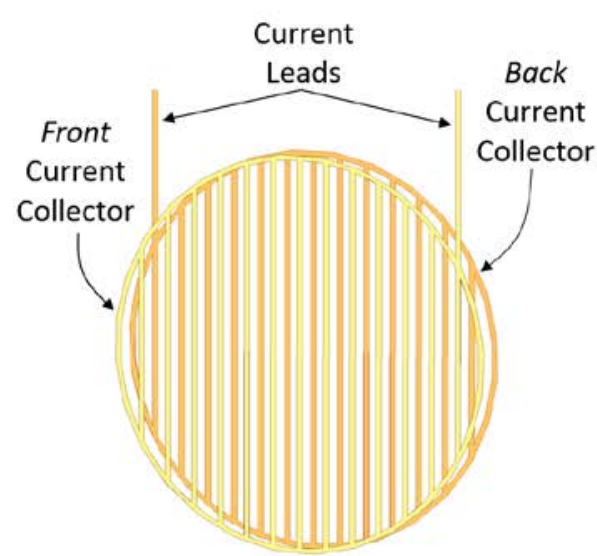

(a)

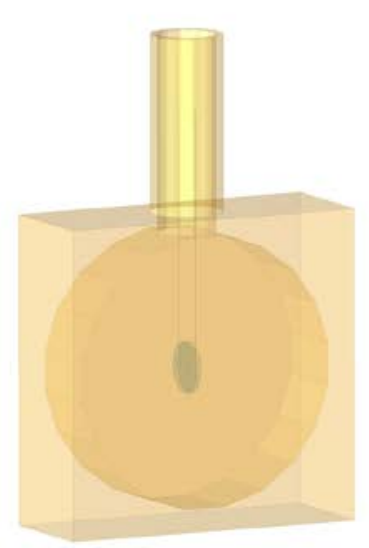

(b)

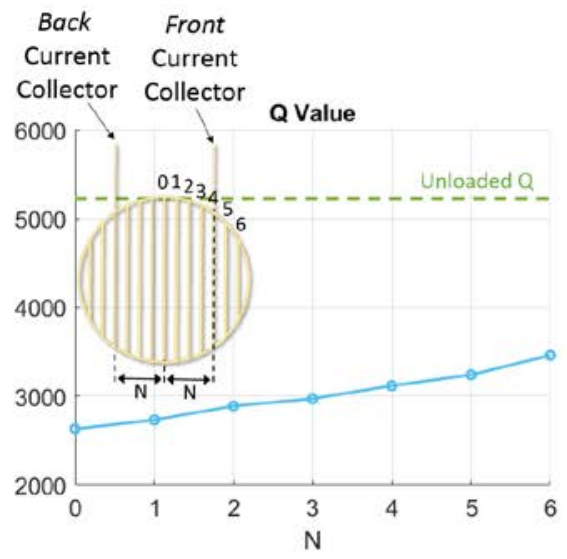

(c)

Figure 7: (a) Sandwiched full-ring current collectors with a line width of $100 \mu \mathrm{m}$. (b) Model of the resonator loaded with sandwiched current collectors. (c) Impact of the position of the current leads on the $Q$ value of the loaded resonator (blue line). The $Q$ value of the unloaded resonator is shown as reference (green dashed line). The inset shows the current collectors. Only the contacting lead is visible for the back current collector, as the rest is concealed by the front current collector.

Table 1: $Q$ Value of the Resonator with Sandwiched Current Collectors

\begin{tabular}{|c|c|c|c|}
\cline { 2 - 4 } \multicolumn{1}{c|}{} & $\begin{array}{c}\text { Quarter-Ring } \\
\text { Configuration }\end{array}$ & $\begin{array}{c}\text { Half-Ring } \\
\text { Configuration }\end{array}$ & $\begin{array}{c}\text { Full-Ring } \\
\text { Configuration }\end{array}$ \\
\hline$Q$ Value & 4200 & 4220 & 3460 \\
\hline
\end{tabular}

An important design parameter is the width of the collectors' lines: they should be as small as possible to prevent shielding of the underlying battery. However, due to manufacturability constraints, the minimum achievable line width currently is $\sim 100 \mu \mathrm{m}$. It can be calculated from the values reported in Table 2 that the metallized area of the full-ring current collector for a line width of $200 \mu \mathrm{m}$ amounts to a prohibitive $\sim 25 \%$ of the total cell area, while a line width of $100 \mu \mathrm{m}$ covers only $\sim 12.5 \%$ of the total cell area. It should be noted that the metallization area required by the full-ring configuration is approximately $122 \%$ of the one required by the quarter-ring and half-ring configurations, therefore the full-ring design should be avoided, unless the diameter of the cell is kept smaller than the one of the ring.

The sandwiched full-ring structure (Figure 7-a) and the model shown in Figure 7-b have been considered again to evaluate the impact of the line width on the measurements. It can be seen from the values listed in Table 2 that, while a line width of $200 \mu \mathrm{m}$ considerably affects the $Q$ value of the loaded resonator, line widths of 50 and $100 \mu$ m have a comparable, more moderate impact on it. 
Table 2: Sandwiched Full-Ring Current Collectors: Required Metallization Area, and Resonator $Q$ Value for Different Line Widths

\begin{tabular}{|c|c|c|c|}
\hline Line Width & $50 \mu \mathrm{m}$ & $100 \mu \mathrm{m}$ & $200 \mu \mathrm{m}$ \\
\hline Metallization Area & $3.1 \mathrm{~mm}^{2}$ & $6.3 \mathrm{~mm}^{2}$ & $12.6 \mathrm{~mm}^{2}$ \\
\hline $\boldsymbol{Q}$ Value & 3267 & 3460 & 1291 \\
\hline
\end{tabular}

Another important factor to take into account is the position of the long current leads shown in Figures 7-a. Figure 7-c shows the impact of the position of the leads with respect to the center of the current collector on the $Q$ value of the resonator. The position is identified by the integer $N$, that represents the alignment of the contact with the $N$-th grating line from the center. The leads on the two current collectors are positioned symmetrically with respect to the center of the cell. The $Q$ value decreases for decreasing $N$, as the long leads of the current collectors on the two sides of the battery get closer to each other and thus eventually act as a transmission line that affects the field distribution within the resonator. Therefore, it is preferable to place these contacts as close as possible to the edges of the cell.

\section{CONCLUSIONS}

We have presented an analysis of the requirements for the manufacture of current collectors suitable for operando EPR measurements of lithium-ion battery cells. Using EM simulations, we have introduced a design procedure that allows a systematic development of the current collectors for circular battery cells.

We have introduced three different grating configurations and evaluated their performance, showing that, depending on the actual size of the active electrodes with respect to the current collectors, a fullring or a half-ring design, connecting vertical lines, would be the most appropriate structure. We have further assessed the impact of the line width and the position of the current leads on the Q value of the loaded EPR cavity and derived recommendations for their dimensioning.

The procedure and guidelines described in this paper can be used for the design of current collectors for arbitrary flat battery cells.

\section{REFERENCES}

1. C. P. Poole, "Electron Spin Resonance - A Comprehensive Treatise on Experimental Techniques", John Wiley \& Sons, $2^{\text {nd }}$ Ed., 1983.

2. J. Wandt, C. Marino, H. A. Gasteiger, P. Jakes, R.-A. Eichel and J. Granwehr, "Operando electron paramagnetic resonance spectroscopy--formation of mossy lithium on lithium anodes during charge-discharge cycling" Energy \& Environmental Science, vol. 8, pp. 1358-1367, 2015.

3. M. Sathiya, J.-B. Leriche, E. Salager, D. Gourier, J.-M. Tarascon and H. Vezin, "Electron paramagnetic resonance imaging for real-time monitoring of Li-ion batteries," Nature Communications, vol. 6, p. 6276, 2015. 
4. J. Wandt, P. Jakes, J. Granwehr, H. A. Gasteiger and R.-A. Eichel, "Singlet oxygen formation during the charging process of an aprotic lithium--oxygen battery," Angewandte Chemie, vol. 128, pp. 70067009, 2016.

5. M. Tang, A. Dalzini, X. Li, X. Feng, P.-H. Chien, L. Song and Y.-Y. Hu, "Operando EPR for Simultaneous Monitoring of Anionic and Cationic Redox Processes in Li-Rich Metal Oxide Cathodes," The Journal of Physical Chemistry Letters, vol. 8, pp. 4009-4016, 2017.

6. A. Niemöller, P. Jakes, S. Eurich, A. Paulus, H. Kungl, R.-A. Eichel and J. Granwehr, "Monitoring local redox processes in LiNi0.5Mn1.5O4 battery cathode material by in operando EPR spectroscopy," The Journal of Chemical Physics, vol. 148, p. 014705, 2018.

7. J. Wandt, P. Jakes, J. Granwehr, R.-A. Eichel and H. A. Gasteiger, "Quantitative and time-resolved detection of lithium plating on graphite anodes in lithium ion batteries," Materials Today, 2017.

8. S. Kayser, A. Mester, A. Mertens, P. Jakes, R. Eichel and J. Granwehr, "Long-run in operando NMR to investigate the evolution and degradation of battery cells," Physical Chemistry Chemical Physics, 2018.

9. D. M. Pozar, "Microwave Engineering", John Wiley \& Sons, $4^{\text {th }}$ Ed., 2012.

10. Bruker Corporation - EPR Imaging. https://www.bruker.com/products/mr/epr/epr-resonatorsacessories/epr-accessories/epr-imaging/overview.html. Retrieved on April, $16^{\text {th }} 2018$. 


\section{1 - Title of the Paper:}

Optimization of Current Collector Design for Operando X-Band-EPR Investigations of Lithium-Ion Batteries using Numerical Simulations

\section{2 - Authors:}

Ivan Flammia ${ }^{1}$, i.flammia@fz-juelich.de

Achim Mester ${ }^{1}$, a.mester@fz-juelich.de

Stefan van Waasen ${ }^{1,4}$, s.van.waasen@fz-juelich.de

Affiliation 1 - Central Institute for Engineering, Electronics and Analytics - Electronic Systems (ZEA2), Forschungszentrum Jülich GmbH, Jülich, Germany

Affiliation 4 - Faculty of Engineering, Communication Systems (NTS)

University of Duisburg-Essen, Duisburg, Germany

Arvid Niemöller², a.niemoeller@fz-juelich.de

Josef Granwehr ${ }^{2,3}$, j.granwehr@fz-juelich.de

Affiliation 2 - Institute of Energy and Climate Research - Fundamental Electrochemistry (IEK-9), Forschungszentrum Jülich GmbH, Jülich, Germany

Affiliation 3 - Institute of Technical and Macromolecular Chemistry (ITMC)

RWTH Aachen University, Aachen, Germany

\section{3 - Mailing Address:}

Ivan Flammia

Central Institute for Engineering, Electronics and Analytics - Electronic Systems (ZEA-2), Forschungszentrum Jülich GmbH

Wilhelm-Johnen-Straße

52428 Jülich

Germany

4 - Phone and Fax Numbers

Phone: +492461 6185093

Fax: +49 2461 61-2175

\section{5 - Corresponding author and Presenting author}

Ivan Flammia is the corresponding author and presenting author.

\section{6 - Topic or Session organizer}

Topic: 6. Resonators, filters, interconnects, packaging, MMIC

\section{7 - State if poster presentation is preferred}

Oral presentation is preferred 